\title{
THE CALCULATION OF ADHESIVE FRACTURE ENERGIES IN MODE I: REVISITING THE TAPERED DOUBLE CANTILEVER BEAM (TDCB) TEST.
}

\author{
B.R.K. BLACKMAN ${ }^{\dagger}$, H. HADAVINIA, A.J. KINLOCH, M. PARASCHI \\ AND J.G. WILLIAMS \\ Department of Mechanical Engineering, Imperial College of Science, Technology and \\ Medicine, Exhibition Road, London SW7 2BX. UK.
}

\begin{abstract}
Analytical corrections have been derived for a beam theory analysis for the adhesively-bonded tapered double cantilever beam (TDCB) test specimen to account for the effects of beam root rotation and for the real, as opposed to idealised, profile of the beam as required experimentally. A number of adhesive-substrate combinations were tested according to a new test protocol and the new analysis method for data reduction is compared critically with the existing simple beam theory and experimental compliance approaches. Correcting the beam theory for root rotation effects is shown to be more important than correcting only for the effects of shear deformation of the substrates. Results from a finite element analysis, using a cohesive zone model, also showed close agreement with the proposed new corrected beam theory (CBT) analysis method.
\end{abstract}

Keywords-adhesive joints, tapered double cantilever beam (TDCB), fracture mechanics, simple beam theory (SBT), corrected beam theory (CBT), root rotation, cohesive zone. 


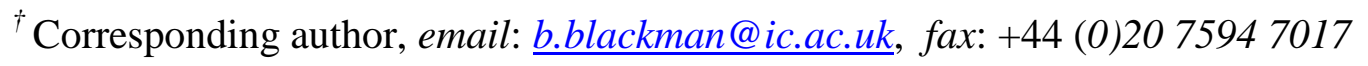

\section{NOMENCLATURE}

\begin{tabular}{|c|c|}
\hline$A_{1,2}$ & integration constants \\
\hline$a$ & crack length \\
\hline$a_{\text {int }}$ & intercept on the crack length axis of a $C$ versus $a$ plot \\
\hline$B_{1,2}$ & integration constants \\
\hline$b$ & width of test specimen \\
\hline C & compliance of the beam \\
\hline E & Young's modulus of the substrate \\
\hline$E_{a}$ & Young's modulus of the adhesive \\
\hline$G_{I C}$ & the adhesive fracture energy \\
\hline$h$ & height of the beam \\
\hline$h_{a}$ & thickness of adhesive layer \\
\hline$h_{o}$ & height of initial, non-profiled section of the TDCB \\
\hline$k$ & the beam foundation stiffness \\
\hline$m$ & specimen geometry factor \\
\hline$\hat{m}$ & a modified specimen geometry factor \\
\hline$P$ & load applied to the test specimen \\
\hline$R$ & ratio of $E / E_{a}$ \\
\hline$u_{o}$ & the load-line displacement of both arms of the specimen during a test \\
\hline$v(x)$ & vertical displacement of the beam at distance $x$ from load-line. \\
\hline$x$ & distance along the beam measured from the load-line \\
\hline$x_{O}$ & length of straight section of the beam measured from the load-line \\
\hline$\delta_{c}$ & a critical separation used in the cohesive zone model \\
\hline
\end{tabular}




$\begin{array}{ll}\Delta & \text { beam root rotation correction } \\ \lambda & \text { ratio of } 2 h / h_{a} \\ \sigma_{\max } & \text { stress parameter used in the cohesive zone model } \\ C B T & \text { corrected beam theory } \\ C Z M & \text { cohesive zone model } \\ \text { DCB } & \text { double cantilever beam } \\ \text { ECM } & \text { experimental compliance method } \\ \text { FE } & \text { Finite element } \\ \text { SBT } & \text { simple shear-corrected beam theory } \\ \text { TDCB } & \text { tapered double cantilever beam }\end{array}$

\section{INTRODUCTION}

The application of fracture mechanics to adhesive joints dates back to the 1960s when Ripling et al [1] and later Mostovoy et al [2] studied experimental methods to determine the plane-strain fracture toughness of bonded metallic joints. This work led to an ASTM standard [3] which used a simple shear-corrected beam theory to deduce the values of $G_{I C}$ from either adhesively bonded double cantilever beam (DCB) or tapered double cantilever beam (TDCB) test specimens. However, this analysis did not consider the effects of beam root rotation, nor did it account for the real, as opposed to idealized, profile of the tapered beam. Indeed, Mostovoy and co-workers [2] noted that the simple shear-corrected beam theory required a correction for rotations at the assumed 'built-in' end of the beam and determined an empirical rotation correction, $a_{o}$, which could be treated as an increase in the crack length. They found experimentally that the correction was approximately equal to $0.6 h$, where $h$ was the height of the beam for the DCB test specimen. However, this correction was not implemented in the 
ASTM standard [3], nor was it suggested how the correction could be used with the height tapered beams employed in the TDCB test specimen.

In the present work, an analytical correction for beam root rotation is derived for use with the tapered double cantilever beam specimen. In addition, the beam theory analysis described considers the actual profile of the test specimen that includes an initial linear section (where the beam height is a constant) as shown in Figure 1. Experimental results obtained from this new, corrected analysis are compared to both the simple shear-corrected beam analysis employed in [3] and also with an experimental compliance calibration method. Results for two different adhesives and two different beam substrate materials, i.e. aluminium alloy and mild steel, are presented. Further, the analytical and experimental results are compared with a finite element analysis employing a cohesive zone model. Finally, the new analysis scheme proposed for the TDCB test has been incorporated into a new test protocol that has been critically assessed in a round-robin programme organized by the European Structural Integrity Society (ESIS), under the Technical Committee on Polymers, Adhesives and Composites. The protocol and initial round-robin results can be found in [4] and a full presentation of the round-robin results will be published shortly [5].

\section{ANALYTICAL STUDIES}

The adhesive fracture energy, $G_{I C}$, for the tapered double cantilever beam adhesive joint specimen may be determined directly using the Irwin-Kies equation, (1):

$$
G_{I C}=\frac{P^{2}}{2 b} \frac{d C}{d a}
$$


where $P$ is the applied load, $b$ the specimen width, $C$ the compliance of the substrate beam (given by the displacement divided by the load) and $a$ is the crack length. The value of $d C / d a$ can be measured experimentally and thus the $G_{I C}$ value calculated. In the present work, equation (1) is described as the experimental compliance method (ECM) of analysis. The analyses that follow all aim to solve for $d C / d a$.

\subsection{Simple shear-corrected beam theory (SBT) analysis}

Mostovoy et al [2] were the first to propose the use of the height tapered double cantilever beam, (TDCB), test geometry for the purpose of measuring the resistance to crack growth in adhesive joints. By first considering a double cantilever beam specimen, the compliance of the beam was determined by considering the contributions from bending and shear deflections. This analysis led to equation (2):

$$
\frac{d C}{d a}=\frac{8}{E b}\left(\frac{3 a^{2}}{h^{3}}+\frac{1}{h}\right)
$$

where $E$ and $h$ are the substrate modulus and height respectively. They proposed that if the height of the beam was carefully profiled, then $d C / d a$ could be held constant by ensuring that the quantity in brackets in equation (2) was also a constant. Thus, if the beam was machined to a geometry factor, $m$, given by:

$$
m=\frac{3 a^{2}}{h^{3}}+\frac{1}{h}=\text { constant }
$$


then the value of $d C / d a$ would be constant, i.e. the compliance would change linearly with crack length and also, by substituting into equations (2) and (1) the adhesive fracture energy was expressed as:

$$
G_{I C}=\frac{4 P^{2}}{E b^{2}} \cdot\left(\frac{3 a^{2}}{h^{3}}+\frac{1}{h}\right)=\frac{4 P^{2}}{E b^{2}} \cdot m
$$

Indeed, equation (4) was applied to either straight, double cantilever beam specimens or height tapered beams as outlined in the ASTM standard [3]. Such specimens have become very popular and are widely used for both static and fatigue testing and also for the ageing of adhesive joints in various environments.

\subsection{Corrected beam theory (CBT) analyses}

\subsubsection{Introduction}

As stated previously, the above analysis considers the deflections of the substrate beams due to bending and shear but does not allow for the important contribution to the compliance from the deflection and rotation at the beam root, i.e. at the assumed built-in crack tip. Indeed, in the present work it is shown that these effects are more important than those associated with shear deformations when predicting the compliance and $G_{I C}$ values of TDCB specimens manufactured with metallic substrates. Equation (3), which defines the geometry factor of the tapered double cantilever beam, is only weakly dependent upon the shear term 1/h. In the analysis that follows, equation (3) has been simplified to equation (5):

$$
m=\frac{3 a^{2}}{h^{3}}
$$


i.e. the shear-correction term $1 / \mathrm{h}$ has been neglected in the calculation of $m$. This simplification allows the bending equations to be readily integrated and enables a simple scheme to be proposed to account for root rotation. For the beams used in the present work, the geometry factor, as defined by equation (3), was always equal to $2 \mathrm{~mm}^{-1}$. Thus, the error in $m$ imposed by the above simplification was $-2 \%$ for a crack length of $100 \mathrm{~mm}$ and $-1.3 \%$ for a crack length of $200 \mathrm{~mm}$, which represents the typical extent of crack propagation observed in the experiments reported here.

\subsubsection{Determining the compliance of the TDCB}

To enable the TDCB specimen to be readily tested, the initial section of the beam is not profiled, thus enabling holes to be drilled through the metallic substrates as shown in Figure 1. Loading pins may then be inserted through the holes and the beam loaded in mode I tension. Beams are manufactured such that the geometry factor $m$, given by equation (3), is a constant. A single arm of the tapered beam is shown in Figure 2 with notation shown. In the present analysis, the height of the straight section of the beam is termed $h_{o}$ and this extends a distance $x_{o}$ from the load-line. The height of the profiled section of the beam is given by $h$ which is a function of the distance $x$, again measured from the load-line. The beam has a width $b$ and a load $P$ and displacement $u_{o} / 2$, is applied at $x=0$ during the test. Because of the discontinuity of the beam profile at $x=x_{0}$, beam theory has been applied to the two sections of the beam separately. Firstly, the straight section of the beam where $0<\mathrm{x}<\mathrm{x}_{\mathrm{o}}$ and secondly the profiled section where $\mathrm{x}>\mathrm{x}_{0}$. If the vertical displacement along the beam is given by $v(x)$, then:

(i) For the straight beam section, $0<\mathrm{x}<\mathrm{x}_{\mathrm{O}}$

From beam theory we can write: 


$$
\frac{d v}{d x}=\frac{4 m}{E b x_{o}^{2}}\left(\frac{P x^{2}}{2}+A_{1}\right)
$$

and

$$
v=\frac{4 m}{E b x_{o}^{2}}\left(\frac{P x^{3}}{6}+A_{1} x+B_{1}\right)
$$

where $A_{1}$ and $B_{1}$ are integration constants. The boundary condition is that at $x=0, v=u_{0} / 2$ where $u_{o}$ is the total displacement of two arms of the TDCB, i.e. the measured displacement during a test. Therefore, from (7):

$$
\frac{u_{o}}{2}=\frac{4 m}{E b x_{o}^{2}} \cdot B_{1}
$$

(ii) For the profiled beam section, $x>x_{O}$

$$
\frac{d v}{d x}=\frac{4 m}{E b}\left(P \ln x+A_{2}\right)
$$

and

$$
v=\frac{4 m}{E b}\left(P x(\ln (x)-1)+A_{2} x+B_{2}\right)
$$


where $A_{2}$ and $B_{2}$ are two further integration constants. The boundary conditions are now the usual built-in beam assumptions, i.e. when $x=a, v=d v / d x=0$, where $a$ is the crack length. This leads to:

$$
A_{2}=-P \ln a \text { and } B_{2}=P a \text {. }
$$

Now, equating the expressions for $v$ and $d v / d x$ at $x=x_{0}$ allows the constants $A_{1}$ and $A_{2}$ to be determined and thus equation (9) becomes:

$$
v=\frac{u_{o}}{2}=\frac{4 m}{E b} \cdot P\left(a-\frac{2}{3} x_{o}\right)
$$

and as we define compliance as $u_{0} / P$, then for $x>x_{0}$ we can write:

$$
C=\frac{8 m}{E b}\left(a-\frac{2}{3} x_{o}\right)
$$

Equation (10) implies that the relationship between compliance and crack length is linear for $x>x_{0}$, as expected, but does not pass through the origin. Indeed, when $C=0$, $a=(2 / 3) x_{o}$ which means that, for a typical beam with $x_{o}=50 \mathrm{~mm}$, a positive $C(a)$ intercept of 33.3mm would be predicted by this bending analysis. The expected variation in compliance with distance $x$ is depicted in Figure 3. For $x<x_{0}$, the compliance is proportional to $x^{3}$, but for $x>x_{0}$, compliance is directly proportional to $x$. Differentiating equation (10) recovers the ASTM equation (2), so whilst identifying the $C(0)$ intercept, equation (10) does not alter the calculation of $G_{I C}$. Equation (10) will be described here as the full-profile SBT analysis method to emphasise that, in its derivation, the full beam profile has been considered. 
However, it is known that the built-in beam assumptions used in this analysis lead to errors in compliance and hence errors in $G_{I C}$. These are now considered.

\subsubsection{Correcting the compliance for beam root rotation}

Various schemes have been reported in the literature for determining the root rotation correction for beam specimens. For example, Kanninen modelled the DCB specimen as a beam on an elastic foundation [6]. Each arm was modelled as a cantilever beam supported on a foundation of stiffness $k$ per unit length. Solving the governing differential equation led to a correction for beam root rotation via $\Delta$. In effect, the crack length, $a$, was replaced by $a+\Delta$, where

$$
\Delta^{4}=\frac{E b h^{3}}{3 k}
$$

where $h$ and $b$ were the height and width respectively of the DCB arm. The foundation stiffness $k$ was deduced by considering the elastic stretching of the foundation, i.e. the beam material. This led to:

$$
\Delta=h\left(\frac{1}{6}\right)^{\frac{1}{4}} \quad \text { i.e. } \quad \Delta \approx 0.64 h
$$

In a later analysis which included the shear deformation of the beam, Kanninen [7] showed that the effects of both shear deflection and beam root rotation could be modelled by taking $\Delta=0.67 \mathrm{~h}$. Williams [8] used a beam on an elastic foundation model to analyse an adhesively-bonded beam specimen. By considering the foundation to be made up of both the 
substrate beam and the adhesive layer, it was shown that the root rotation, $\Delta$, could be expressed as:

$$
\Delta=h\left(\frac{1}{6}\right)^{\frac{1}{4}}\left(1+\frac{h_{a}}{2 h} \frac{E}{E_{a}}\right)^{\frac{1}{4}}
$$

where $h$ and $E$ are the height and Young's modulus of the substrate respectively, and $h_{a}$ and $E_{a}$ are the thickness and Young's modulus of the adhesive layer respectively. Equation (13) may be more conveniently expressed as:

$$
\Delta=h\left(\frac{1}{6}\right)^{\frac{1}{4}}\left(1+\frac{R}{\lambda}\right)^{\frac{1}{4}}
$$

Where $\lambda=2 h / h_{a}$ and $R=E / E_{a}$. Clearly, the contribution of the adhesive layer to $\Delta$ depends upon the values of $\lambda$ and $\mathrm{R}$. In the present work, $\Delta$ has been obtained via equation (12). The errors introduced by this simplification were calculated and are discussed in section 5.5 . Thus, by using the root rotation correction as deduced from equation (12), and again making the simplifying assumption for the geometry factor $m$, i.e. by using equation (5) rather than equation (3), $\Delta$, can be simply expressed as:

$$
\Delta=0.64\left(\frac{3}{m}\right)^{\frac{1}{3}} \cdot a^{\frac{2}{3}}
$$

Now, replacing $a$ with $(a+\Delta)$ in equation (10), and substituting for $\Delta$ from equation (15) leads to: 


$$
C=\frac{8 m}{E b}\left(a+0.64\left(\frac{3}{m}\right)^{\frac{1}{3}} \cdot a^{\frac{2}{3}}-\frac{2}{3} x_{o}\right)
$$

Finally, differentiating equation (16) and substituting into equation (1) yields the following expression for $G_{I C}$ :

$$
G_{I C}=\frac{4 P^{2} m}{E b^{2}}\left(1+0.43\left(\frac{3}{m a}\right)^{\frac{1}{3}}\right)
$$

In the present work, equations (16) and (17) are described as the corrected beam theory (CBT) analysis equations for the tapered double cantilever beam specimen. This analysis has been employed in the new test protocol [9] and has been critically examined during round-robin testing [4].

\subsubsection{Correcting for shear and root rotation via $m$}

As a possible alternative to the new analytical corrections proposed above, the geometry of the TDCB could be modified to incorporate corrections for root rotation and shear. The analyses developed in $[6,7,10]$ could be employed to identify a new geometry factor, $\hat{m}$, such that:

$$
\hat{m}=\frac{3(a+0.67 h)^{2}}{h^{3}}=\frac{3 a^{2}}{h^{3}}+\frac{4 a}{h^{2}}+\frac{1.35}{h}=\text { constant }
$$

Such a modified TDCB specimen could then be defined in which the substrate beams were profiled such that the value of $\hat{m}$ was held constant. However, due to the widespread 
popularity of the original TDCB geometry based upon equation (3), the present authors have considered that the correction scheme outlined in sections 2.2.2-2.2.3 is a better approach and thus no modification has been made to the beam profile in the present work.

In the following sections, the accuracy of equations (4) and (17) have been assessed by direct comparison with the Irwin-Kies equation (1) and also for one joint system, with a finite element analysis employing a cohesive zone model, as will now be described.

\section{FINITE ELEMENT (FE) ANALYSIS STUDIES}

The tapered double cantilever beam test has been analysed numerically using a cohesive zone model (CZM) [11]. In the analysis, the variation of cohesive stress as a function of interfacial opening is defined along the process zone local to the crack tip. The method has been widely used to predict the global failure by introducing the local fracture parameters, i.e. the fracture energy $G_{C}$ and a stress parameter, $\sigma_{\max }$ [11-13].

In the present work, cohesive elements based upon a traction-separation law have been introduced along the crack path at the centre of the adhesive layer assuming a cohesive fracture, as observed in the experiments. This resulted in a continuum description of the failure path. A cubic traction-separation law (see Figure 4) was used [14], based on the adhesive fracture energy $G_{I C}$ and the stress parameter, $\sigma_{\max }$. The value of $\sigma_{\max }$ was taken to be equivalent to the uniaxial yield stress of the adhesive, i.e. 50MPa. However, the shape of the traction-separation is of secondary importance [15]. Once the shape is fixed, the critical separation, $\delta_{c}$, is no longer an independent parameter. When separation takes place under both normal and tangential crack face displacements, a potential can be used to generate the relation between the traction components and the displacements such that the work of 
separation remains equal to $G_{C}$. The cohesive zone model was incorporated into the finite element code ABAQUS (version 5.8) via a user subroutine.

\section{EXPERIMENTAL STUDIES}

\subsection{Joint manufacture}

Bonded tapered double cantilever beams were manufactured using substrates consisting of either aluminium alloy (grades ENAW5083 or ENAW2014A) or mild steel (grade EN32b). Two rubber toughened, structural adhesives were employed; an epoxy-paste adhesive (ESP110 from Permabond, UK) and an epoxy-film adhesive (AF126 from 3M, USA). Beams were manufactured using a CNC milling machine to produce a constant geometry factor, $m=2 \mathrm{~mm}^{-1}$ as defined in [3] and given by equation (3). The length of the straight section, $x_{o}$, was $50 \mathrm{~mm}$. All substrates were surface treated such that all crack propagation occurred cohesively in the adhesive layer. The bond-line thickness of the epoxypaste adhesive was controlled during manufacture using $0.4 \mathrm{~mm}$ diameter wire inserts. When bonding with the epoxy-film adhesive, a single layer of film was used which produced a final bond-line thickness of about $0.15 \mathrm{~mm}$. The adhesive joints were cured according to the manufacturers' instructions. A PTFE insert film of thickness 12.5 microns was inserted into the adhesive layer at the loading end to create an initial crack. Typically this extended 100mm from the load-line.

\subsection{Joint testing}

A series of tests were performed following the new protocol [9]. Loading was carried out at a constant displacement rate of $0.1 \mathrm{~mm} / \mathrm{min}$. Values of the load, $P$, the displacement $u_{o}$ and the crack length, $a$, were measured for approximately $100 \mathrm{~mm}$ of crack propagation, i.e. approximately from $x=100$ to $x=200 \mathrm{~mm}$. Full unloading of the joints prior to catastrophic 
failure was always conducted to ensure that the conditions of LEFM were not violated. In all cases, only elastic deformation of the substrates occurred. The compliance of the tensile loading system (including all the pins and shackles) was measured by attaching a very stiff calibration specimen in place of the test specimen and loading to the maximum load attained in the fracture test. All displacement values recorded from the fracture tests were then corrected for the system compliance.

\section{RESULTS AND DISCUSSION}

\subsection{Aluminium alloy substrates bonded with the epoxy-paste adhesive}

Experimental data from two repeat tests are shown in Figure 5. The values of compliance, $C$, have been plotted against crack length, $a$. When plotting these data, only values of compliance associated with crack propagation were used, i.e. all crack initiation points were excluded from the analysis. The experimental data are shown as the unfilled points. Also shown in Figure 5 are the values of $C$ determined from simple beam theory (SBT), i.e. from the equation $C=8 \mathrm{ma} / \mathrm{Eb}$. In addition, the values of $C$ have been determined using full-profile SBT, i.e. by using equation (10). It should be recalled that full-profile SBT considers the actual geometry of the beam used, i.e. with the initial non-profiled section, but does not include the correction for root rotation. The parameters used in the analysis of these data were:

$$
b=9.83 \mathrm{~mm}, E=71 \mathrm{GPa}, h_{a}=0.4 \mathrm{~mm}, x_{o}=50 \mathrm{~mm}, h_{o}=16 \mathrm{~mm} \text { and } m=2 \mathrm{~mm}^{-1} .
$$

From Figure 5, the following observations can be made. Firstly, the $C$ versus $a$ data from the two experimental tests performed were very repeatable. A single linear regression 
line has been drawn on Figure 5 through these data, but the results of the individual regression analyses for the two tests respectively are shown in Table I. These data were highly correlated with a correlation coefficient, $\mathrm{r}^{2}=0.999$ or greater, and an intercept with the crack length axis at $a=17 \pm 0.6 \mathrm{~mm}$. Secondly, the values of $C$ versus $a$ calculated using SBT, i.e. by assuming $C=8 m a / E b$, and the values determined using full-profile SBT, i.e. equation (10), yielded lines with the same slope but different intercepts. These lines intercept the crack length axis, $a_{\text {int }}$ at zero and $33.3 \mathrm{~mm}$ respectively. The slopes of these analytical lines are lower than that measured experimentally, i.e. the analytical value of $d C / d a$ via SBT or fullprofile SBT is lower than the experimental value as shown in Table I. This observation leads to values of $G_{I C}$ deduced via equation (4) being significantly lower than values deduced via equation (1), which uses the experimentally determined values of $d C / d a$. Finally, when extrapolating the $C$ versus $a$ values back to the $C=0$ axis as shown in Figure 5, it is important to remember that all the experimental data were obtained from the profiled section of the beam and hence the extrapolated lines shown in the figure are a fit to these data.

Figure 6 shows the previous experimental data from Figure 5 but the values of the compliance predicted by the corrected beam theory (CBT), i.e. equation (16), and also the values predicted by the finite element cohesive zone model (FE-CZM) are also included. It should be noted that both of these approaches allow for root rotation effects. It can be seen that the CBT analysis is a much better fit to the experimental data than was achieved using either the SBT or full-profile SBT analyses. The results produced by CBT and the FE-CZM approaches are summarised in Table I. The results from the FE-CZM approach show good agreement with the CBT analysis.

Figure 7 shows the resistance curve (R-curve) for one test, (Test 1), in which the values of $G_{I C}$ are plotted against crack length. The values of $G_{I C}$ shown in Figure 7 are all 
associated with crack propagation. The adhesive fracture energy has been calculated using the: (a) SBT, i.e. equation (4); (b) CBT, i.e. equation (17); and (c) ECM, i.e. equation (1) approaches. Clearly, the SBT approach is rather conservative, lying $7 \%$ below the values calculated using equation (1). On the other hand, the CBT approach is more accurate (within $2 \%$ of the experimental compliance method (ECM)) but is non-conservative in this case (it should be noted that the $G_{I C}$ axis scale in Figure 7 has been expanded for clarity and does not start at zero). The above observations demonstrate that the CBT analysis predicts the compliance of the TDCB specimen more accurately than either the SBT or full-profile SBT, and leads to an improved accuracy in the values of $G_{I C}$ calculated.

\subsection{Aluminium alloy substrates bonded with the epoxy-film adhesive}

The aluminium alloy used to manufacture these joints was grade 'ENAW 2014A.' The adhesive employed was the single part epoxy-film formulation and a single layer of adhesive was applied to make the joints. The parameters for the analysis of these joints were:

$$
b=9.83 \mathrm{~mm}, E=74 \mathrm{GPa}, h_{a}=0.15 \mathrm{~mm}, x_{o}=50 \mathrm{~mm}, h_{o}=16 \mathrm{~mm} \text { and } m=2 \mathrm{~mm}^{-1}
$$

Tests were performed exactly as before, according to the protocol [9]. The linear regression analyses performed on the experimental data are summarised in Table II. The values of $d C / d a$ predicted by full-profile SBT and CBT, and the values of $a_{i n t}$ are also shown in the table. As before, the regression analysis for SBT has the same slope as full-profile SBT, and passes through the origin. It can be seen that CBT predicts the experimental value of $d C / d a$ with high accuracy, and hence the agreement between values of $G_{I C}$ calculated via the experimental compliance method, i.e. equation (1), are in excellent agreement with the values calculated using the corrected beam theory of equation (17), as shown in Figure 8. 
Figure 8 shows the R-curve for one test, (Test 1), with values of $G_{I C}$ calculated using the SBT, CBT and ECM analysis methods, (it should be noted that the $G_{I C}$ axis scale in Figure 8 has been expanded for clarity and does not start at zero). Again, SBT is conservative and is, on average, 13\% below ECM. The CBT analysis however, is just 4\% below ECM for Test 1, and was within $1 \%$ for Test 2.

\subsection{Mild steel substrates bonded with the epoxy-paste adhesive}

These joints were manufactured using mild steel substrates (grade EN32b). The epoxy-paste adhesive was employed to bond these joints. The parameters for the analysis of these joints were:

$$
b=10.0 \mathrm{~mm}, E=207 \mathrm{GPa}, h_{a}=0.4 \mathrm{~mm}, x_{o}=50 \mathrm{~mm}, h_{o}=16 \mathrm{~mm} \text { and } m=2 \mathrm{~mm}^{-1}
$$

It was apparent from testing these joints that the measured experimental data (i.e. the $C$ versus $a$ data) were subject to a greater degree of variation than had been observed when using the aluminium alloy as substrates. The measured data showed greater variation in both the slope $(d C / d a)$ and the intercept $\left(a_{i n t}\right)$ than was observed for the joints manufactured with aluminium alloy substrates. The mean and standard deviation values of $d C / d a, a_{i n t}$ and correlation coefficient $r^{2}$, are shown in Table III for four tests. The values of $d C / d a$ and $a_{\text {int }}$ predicted by full-profile SCB and CBT are summarised in the table.

From the results in Table III it is clear that CBT agrees more closely with the experimental average than full-profile SBT. However, the experimental data were more scattered, showing comparatively large variations in the linear regression parameters obtained, as indicated by the standard deviations shown in the table. This observation was 
also noted in the results of the inter-laboratory round-robin programme [5]. The mean propagation values of $G_{I C}$ from the four tests conducted on the joints consisting of mild steel substrates bonded with the epoxy-paste adhesive are shown in Table IV, together with the standard deviations obtained. These values are simply the mean of all the propagation values recorded via each analysis method.

The reason for the greater degree of scatter noted in these experimental data is likely to stem from the smaller beam opening displacement values, $u_{o}$, which are measured when the stiffer, mild steel substrates were used. The maximum displacement values required to extend the crack by $100 \mathrm{~mm}$ was about $2.6 \mathrm{~mm}$ when mild steel substrates were used and was about $3.6 \mathrm{~mm}$ when the aluminium alloy substrates were used with the epoxy-paste adhesive. Although the test data were all corrected for machine compliance effects, the values of $u_{o}$ were determined from the crosshead travel of the tensile testing machine. Thus, the smaller displacements occurring during the testing of mild steel joints led to greater errors in the measured $C$ values and consequently, to greater variation in the experimental values of $d C / d a$ and $a_{\text {int }}$ as was shown in Table III. This observation is in line with the trend in the agreement between the CBT and ECM approaches, namely that joints requiring larger displacement values to obtain crack propagation show closer agreement between equations (17) and (1).

\subsection{A comparison of non-dimensionalised compliance values}

It is apparent from equations (10) and (16) that the product $C E b$, depends only upon the geometry of the beam, (i.e. upon the geometry factor, $m$ and the distance $x_{o}$ ) and on the crack length, $a$. Thus, measured values of $C E b$ should be independent of the substrate material employed. The variation in values of $C E b$ with $a$ have been determined for each adhesive joint system tested and one example data set for each joint is shown in Figure 9. Also shown are the values of $C E b$ predicted by the CBT analysis, i.e. by rearranging (16). It 
can be seen that the experimental values of $C E b$ obtained from three different adhesive joint systems are all in close agreement with the values predicted by the CBT analysis. The joints consisting of mild steel substrates showed greater variations in the values of $C E b$, as would be expected from the earlier observations.

\subsection{The accuracy of the root rotation correction}

As stated previously, in the present work the root rotation term, $\Delta$, was calculated using equation (12), which does not consider the adhesive layer in its derivation. Table V compares the values of $\Delta$, for the three adhesive joint systems employed in the present work, calculated using equations (12) and (14). Equation (14) accounts for the presence of both the adhesive layer and the substrate beam. The percentage error in $\Delta$ introduced by ignoring the adhesive layer is also shown in the table. The values for the Young's modulus of the adhesives, $E_{a}$, were $4 \mathrm{GPa}$ and 2.58GPa for the epoxy-paste and epoxy-film adhesives respectively. Thus, the values of $R$, i.e. $\left(E / E_{a}\right)$ for the three adhesive joint systems, in the order presented were: 17.75, 28.68 and 51.75.

Table $\mathrm{V}$ shows the values of $\Delta$ at three different crack lengths approximately corresponding to the start, middle and end of each test respectively. Clearly, the greatest error in $\Delta$ is observed for the mild steel substrates, which possess the greatest value of $R$. The smallest error in $\Delta$ was observed for the aluminium alloy substrates when bonding the epoxyfilm adhesive. Thus, the use of equation (12) leads to an under correction of beam theory and it has been calculated [16] that this leads to an error in $G_{I C}$ of about $1 \%$ for the joints manufactured with the aluminium alloy substrates and about $1.7 \%$ for joints manufactured using the mild steel substrates. These relatively small errors demonstrate the acceptability of 
neglecting the contribution of the adhesive layer to the root rotation term, $\Delta$, for commonly employed joint systems.

\subsection{The Effect of Residual Stress}

In the preceding analysis no account has been taken of residual stress that may arise as the joints cool from the cure temperature during manufacture. These stresses stem from the mismatch of thermal expansions between the substrate and the adhesive or from the chemical shrinkage of the adhesive. The magnitude of the residual stresses introduced also depends upon the cure temperature of the adhesive. The higher the cure temperature, the greater the residual stresses become.

Nairn considered the effects of residual stress upon the measured values of $G_{I C}$ for adhesive joints manufactured using the double cantilever beam geometry [17]. In a more recent analysis [16] the tapered double cantilever beams considered in the present work were investigated for residual stress effects. The results demonstrated that, because of the relatively large values of $h$ employed in the TDCB profile and the modest cure temperatures that was used to manufacture these joints, the residual stresses caused the calculated values of $G_{I C}$ to be about $1 \%$ too high when the aluminium alloy substrates were employed and about $0.65 \%$ too high when the mild steel substrates were used. The residual stress effects were thus relatively very minor for the results presented in the present work. However, residual stress effects may become more important when the DCB test geometry is used, where the value of $h$ is usually lower. Finally, the residual stresses will, of course, change if the adhesive thickness or adhesive modulus changes, and would usually be greater for adhesives cured at higher temperatures.

\section{CONCLUSIONS}


A new corrected beam theory (CBT) analysis, embodied in equations (16) and (17), has been derived for the tapered double cantilever beam (TDCB) adhesive joint specimen. This analysis takes into account the discontinuous profile of the beam and proposes a simple scheme to correct for beam root rotation effects. It has been demonstrated that a previously published [1-3] simple shear-corrected beam theory analysis leads to errors in the calculated compliance and also to the calculation of overly conservative values of adhesive fracture energy, $G_{I C}$. The new analysis has been compared with results obtained using an experimental compliance method and also using a finite element analysis approach employing a cohesive zone model. Close agreement in the results from these two approaches and the new CBT analysis was observed. The various simplifications in the new analysis have been shown to introduce only small errors when compared to the errors incurred by neglecting the effects of beam root rotation and by not considering the real profile of the beam. This new corrected beam theory approach is now embodied in the new British Standard [9].

Acknowledgement---The authors wish to thank the EPSRC (Advanced Fellowship AF/992781) and the National Physical Laboratory for financial support. For performing the residual stress calculations, the authors wish to thank Professor John Nairn at the University of Utah. For providing a valuable discussion forum, the authors wish to thank the European Structural Integrity Society’s Technical Committee (4) on Polymers, Adhesives and Composites.

\section{REFERENCES}

1. Ripling, E.J., S. Mostovoy, and R.L. Patrick, Measuring fracture toughness of adhesive joints. Materials Research \& Standards (ASTM Bulletin), 1964. 4(3, March): p. $129-134$. 
2. Mostovoy, S., P.B. Crosley, and E.J. Ripling, Use of crack-line loaded specimens for measuring plane-strain fracture toughness. Journal of Materials, 1967. 2(3): p. 661681.

3. ASTM, ASTM D3433, in Annual book of ASTM standards. Adhesives section 15. 1990: Philadelphia.

4. Blackman, B.R.K. and A.J. Kinloch, Fracture tests on structural adhesive joints, in Fracture mechanics testing methods for polymers, adhesives and composites, D.R. Moore, A. Pavan, and J.G. Williams, Editors. 2001, Elsevier Science Ltd.: Amsterdam. p. 225-267.

5. Blackman, B.R.K. and A.J. Kinloch, Measuring the Mode I adhesive fracture energy of structural adhesive joints: The results of a European round-robin. to be published, 2001.

6. Kanninen, M.F., An augmented double cantilever beam model for studying crack propagation and arrest. International Journal of Fracture, 1973. 9(1): p. 83-92.

7. Kanninen, M.F., A dynamic analysis of unstable crack propagation and arrest in the DCB test specimen. International Journal of Fracture, 1974. 10(3): p. 415-431.

8. Williams, J.G. Fracture in adhesive joints: The beam on elastic foundation model. in Proc. International Mechanical Engineering Congress \& Exhibition. ASME Symposium on Mechanics of Plastics and Plastics Composites. 12-17 November 1995. San Francisco, USA.

9. Blackman, B.R.K. and A.J. Kinloch, Determination of the mode I adhesive fracture energy, $G_{I C}$, of structural adhesives using the double cantilever beam (DCB) and the tapered double cantilever beam (TDCB) specimens. ESIS TC4 Protocol, 2000 and British Standard BS 7991-2001.

10. Williams, J.G., End corrections for orthotropic DCB specimens. Composites Science and Technology, 1989. 35: p. 367-376. 
11. Tvergaard, V. and J.W. Hutchinson, The relation between crack growth resistance and fracture process parameters in elastic-plastic solids. Journal of Mechanics and Physics of Solids, 1992. 40: p. 1377-1397.

12. Tvergaard, V. and J.W. Hutchinson, The influence of plasticity on mixed mode interface toughness. Journal of Mechanics and Physics of Solids, 1993. 41: p. 11191135.

13. Needleman, A.A., A continuum model for void nucleation by inclusion debonding. Journal of Applied Mechanics, 1987. 54: p. 525-531.

14. Busso, E., Chen, J., Crisfield, M., Kinloch, A.J., Matthews, F.L., and Qui, Y., Predicting progressive delamination of composite material specimens via interface elements. Mechanics of Composite Materials and Structures, 1999. 6, p301-318.

15. Williams, J.G. and H. Hadavinia, Analytical solution of cohesive zone models. Journal of Mechanics and Physics of Solids (in press), 2001.

16. Nairn, J.A., Personal communication. 2000.

17. Nairn, J.A., Energy release rate analysis for adhesive and laminate double cantilever beam specimens emphasizing the effect of residual stresses. International Journal of Adhesion and Adhesives, 1999. 20: p. 59-70. 
Table I. Results of the linear regression analyses for aluminium alloy substrates bonded with the epoxy-paste adhesive.

\begin{tabular}{lccc}
\hline & $a_{\text {int }}(\mathrm{mm})$ & $d C / \mathrm{da}\left(\mathrm{N}^{-1}\right)$ & $r^{2}$ \\
\hline Test 1 & 17.6 & $2.46 \times 10^{-5}$ & $0.9990^{\mathrm{a}}$ \\
Test 2 & 16.5 & $2.47 \times 10^{-5}$ & $0.9997^{\mathrm{a}}$ \\
SBT: $(C=8 \mathrm{ma} / \mathrm{Eb})$ & 0 & $2.29 \times 10^{-5}$ & 1.0 \\
full-profile SBT: eqn. (10) & 33.3 & $2.29 \times 10^{-5}$ & 1.0 \\
CBT: eqn. (16) & 26.5 & $2.50 \times 10^{-5}$ & 1.0 \\
FE-CZM & 20.1 & $2.38 \times 10^{-5}$ & 1.0 \\
\hline
\end{tabular}

$\left({ }^{\mathrm{a}}\right)$ Initiation values from the tests are not included in the regression analyses. 
Table II. Results of the linear regression analysis for aluminium alloy substrates bonded with the epoxy-film adhesive.

\begin{tabular}{lccc}
\hline & $a_{\text {int }}(\mathrm{mm})$ & $d C / d a\left(\mathrm{~N}^{-1}\right)$ & $r^{2}$ \\
\hline Test 1 & 25.8 & $2.61 \times 10^{-5}$ & $0.9986^{\mathrm{a}}$ \\
Test 2 & 21.6 & $2.38 \times 10^{-5}$ & $0.9965^{\mathrm{a}}$ \\
full-profile SBT: eqn. (10) & 33.3 & $2.17 \times 10^{-5}$ & 1.0 \\
CBT: eqn. (16) & 26.5 & $2.38 \times 10^{-5}$ & 1.0 \\
\hline
\end{tabular}

$\left({ }^{a}\right)$ Initiation values from the tests are not included in the regression analyses. 
Table III. Results of the linear regression analysis for mild steel substrates bonded with the epoxy-paste adhesive.

\begin{tabular}{lccc}
\hline & $a_{\text {int }}(\mathrm{mm})$ & $d C / d a\left(\mathrm{~N}^{-1}\right)$ & $r^{2}$ \\
\hline Experimental $^{b}$ & $10.0 \pm 15.3$ & $8.77 \pm 1.22 \times 10^{-6}$ & $0.9886 \pm 0.01^{\mathrm{a}}$ \\
full-profile SBT: eqn. (10) & 33.3 & $7.73 \times 10^{-6}$ & 1.0 \\
CBT: eqn. (16) & 26.5 & $8.51 \times 10^{-6}$ & 1.0 \\
\hline
\end{tabular}

$\left({ }^{a}\right)$ Initiation values from the tests are not included in the regression analyses. $\left(^{b}\right)$ The $( \pm)$ indicate the standard deviations obtained from four repeat tests. 
TABLE IV. Values of $G_{I C}$ obtained from four repeat tests on joints consisting of mild steel substrates bonded with the epoxy-paste adhesive.

\begin{tabular}{lccc}
\hline & & $G_{I C}\left(\mathrm{~J} / \mathrm{m}^{2}\right)$ \\
Analysis method & $S B T$ (eqn. 4) & $C B T$ (eqn. 17) & ECM (eqn. 1) \\
\hline Mean value & 930 & 1019 & 1060 \\
Standard deviation & 56 & 62 & 190 \\
\hline
\end{tabular}


Table V. The root rotation term $\Delta$ calculated using equations (12) and (14) for the adhesive joints used in the present study, and the \% error in $\Delta$.

\begin{tabular}{|c|c|c|c|c|}
\hline Adhesive joint & $\lambda$ & $\Delta$ eqn. (12) & $\Delta$ eqn. (14) & \% Error \\
\hline & \multicolumn{4}{|c|}{$($ For $a=101 \mathrm{~mm}, h=24.99 \mathrm{~mm})$} \\
\hline Al-alloy/epoxy-paste & 119.02 & 15.97 & 16.53 & 3.42 \\
\hline Al-alloy/epoxy-film & 333.25 & 15.97 & 16.30 & 2.04 \\
\hline \multirow[t]{2}{*}{ Mild steel/epoxy-paste } & 119.02 & 15.97 & 17.48 & 8.63 \\
\hline & \multicolumn{4}{|c|}{$($ For $a=150 \mathrm{~mm}, h=32.48 \mathrm{~mm})$} \\
\hline Al-alloy/epoxy-paste & 154.79 & 20.76 & 21.33 & 2.68 \\
\hline Al-alloy/epoxy-film & 433.12 & 20.76 & 21.09 & 1.59 \\
\hline \multirow[t]{2}{*}{ Mild steel/epoxy-paste } & 154.69 & 20.76 & 22.31 & 6.96 \\
\hline & \multicolumn{4}{|c|}{$($ For $a=200 \mathrm{~mm}, h=39.32 \mathrm{~mm})$} \\
\hline Al-alloy/epoxy-paste & 187.22 & 25.12 & 25.70 & 2.24 \\
\hline Al-alloy/epoxy-film & 524.21 & 25.12 & 25.46 & 1.32 \\
\hline Mild steel/epoxy-paste & 187.22 & 25.12 & 26.70 & 5.92 \\
\hline
\end{tabular}




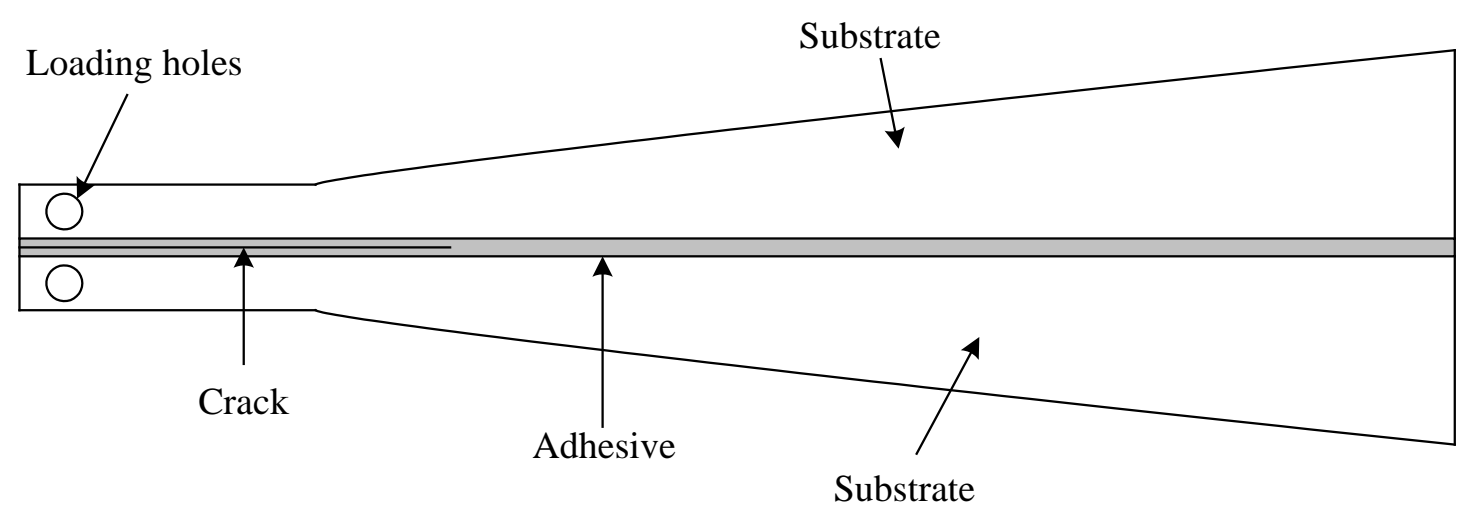

Figure 1. The tapered double cantilever beam (TDCB) adhesive joint test specimen. 


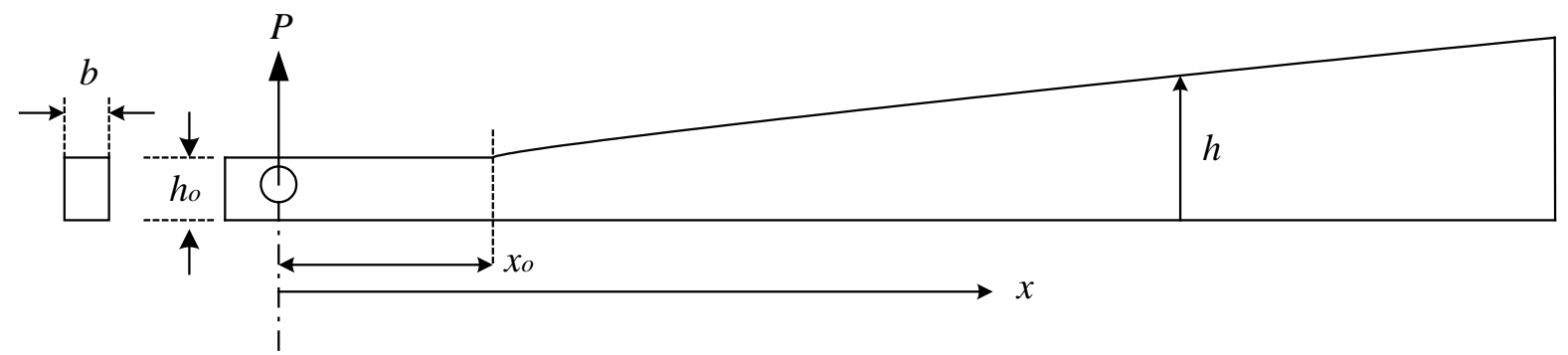

Figure 2. Single arm of the TDCB test specimen without adhesive, showing notation and loading. 


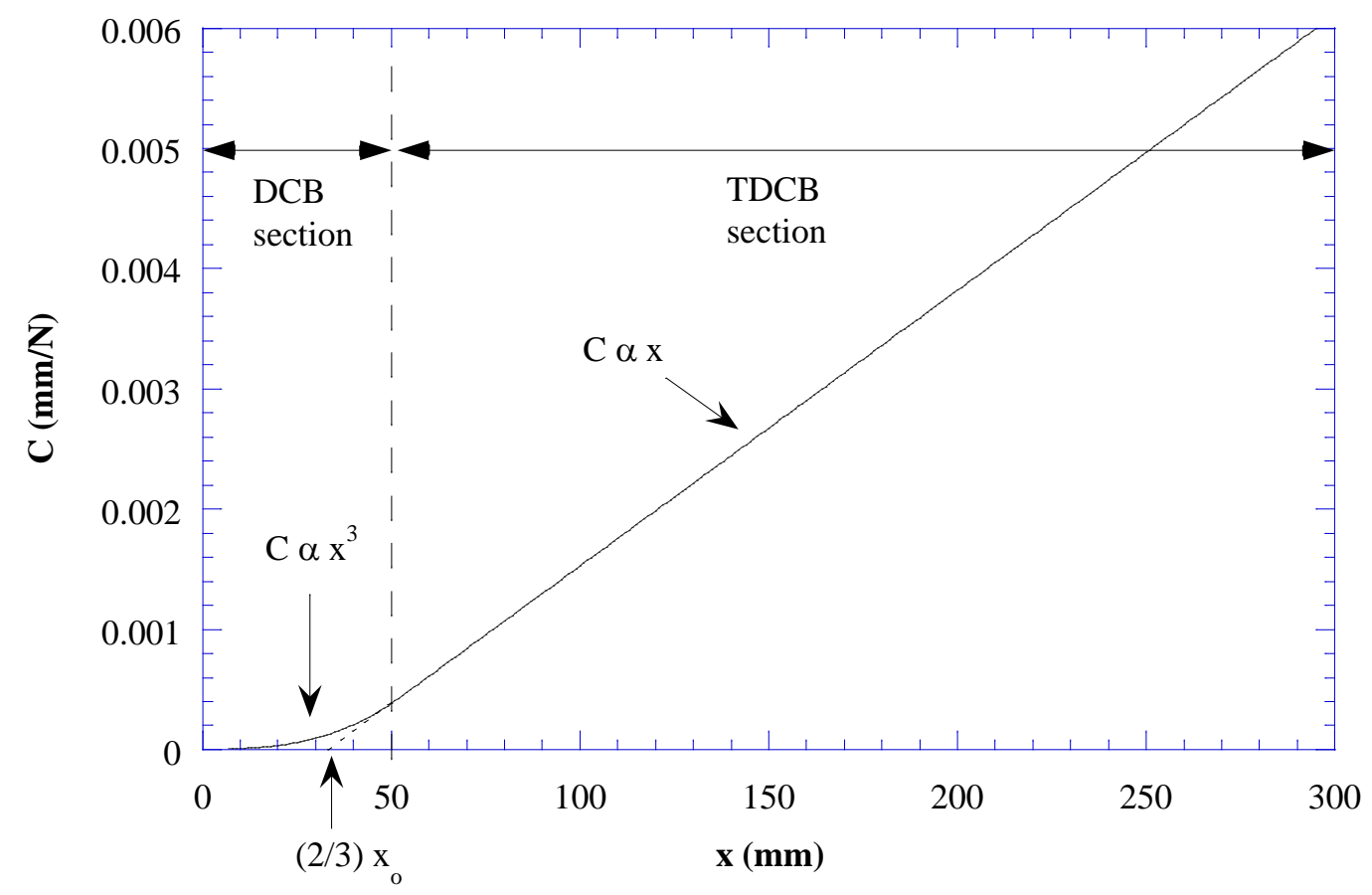

Figure 3. The variation in compliance, $C$, with crack position, $x$, for a tapered double cantilever beam specimen with $x_{o}=50 \mathrm{~mm}, h_{o}=16 \mathrm{~mm}, E=71 \mathrm{GPa}, \mathrm{m}=2 \mathrm{~mm}^{-1}$ and $b=9.81 \mathrm{~mm}$. The initial straight part of the beam is denoted as the DCB section, and the height profiled part is denoted as the TDCB section. (Solid line is equation (10), the dashed line is the extrapolation of the linear TDCB section back to $C=0$.) 


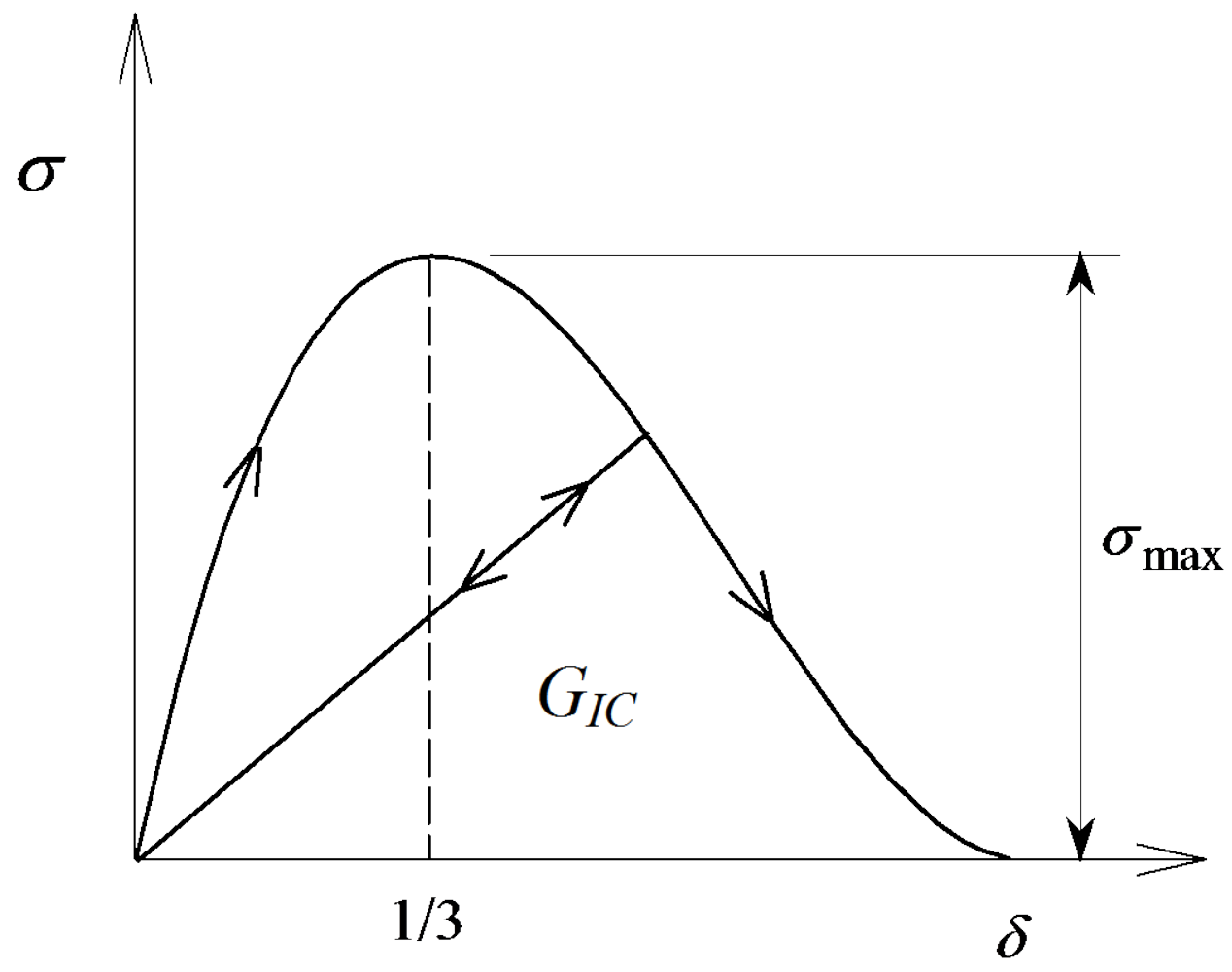

Figure 4. The cubic traction-separation law employed in the cohesive zone model analysis. 


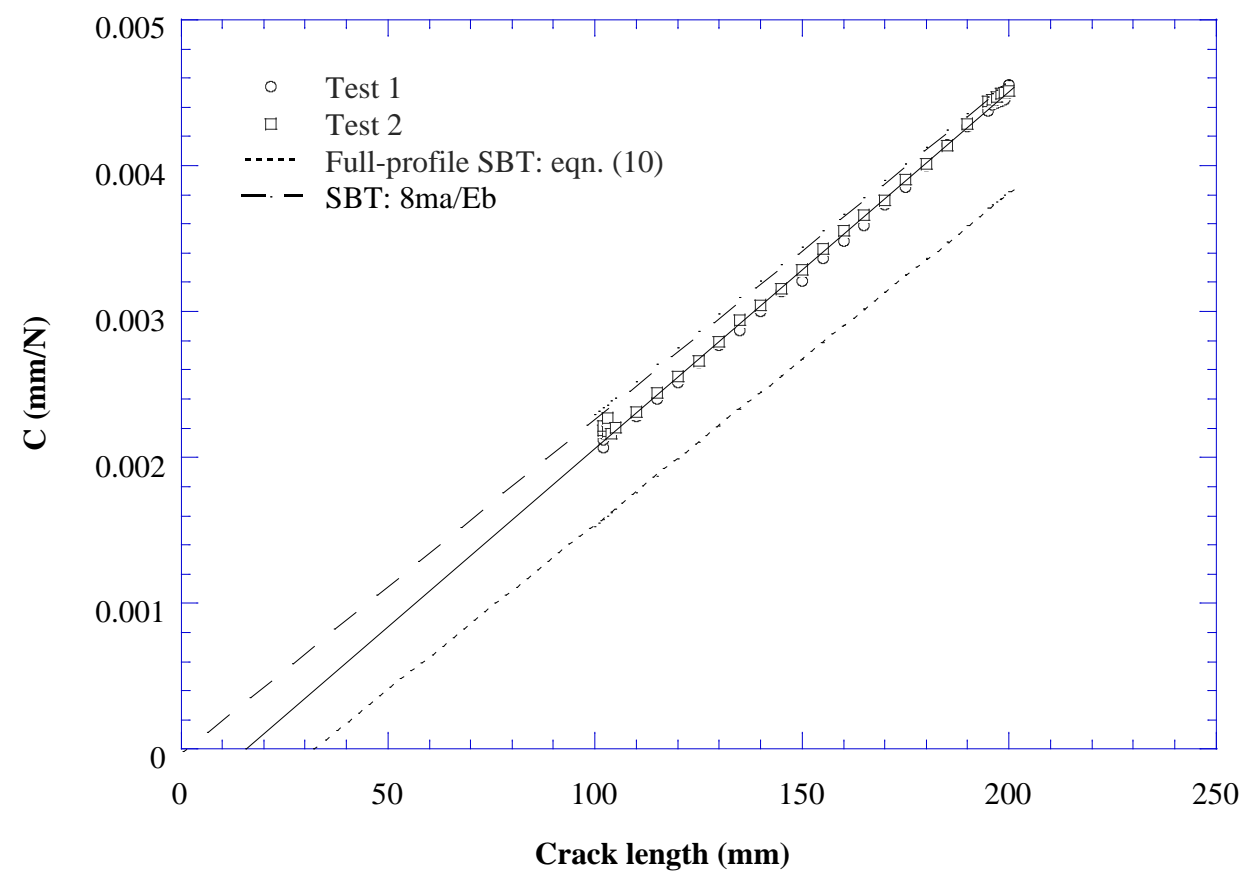

Figure 5. The variation of compliance with crack length for a tapered double cantilever beam specimen manufactured with aluminium alloy substrates and bonded with the epoxy-paste adhesive. Experimental results from two tests are shown, together with the values predicted by the full-profile SBT, i.e. equation (10), and from SBT, i.e. $C=8 m a / E b$. The solid line is a regression line through the experimental data. 


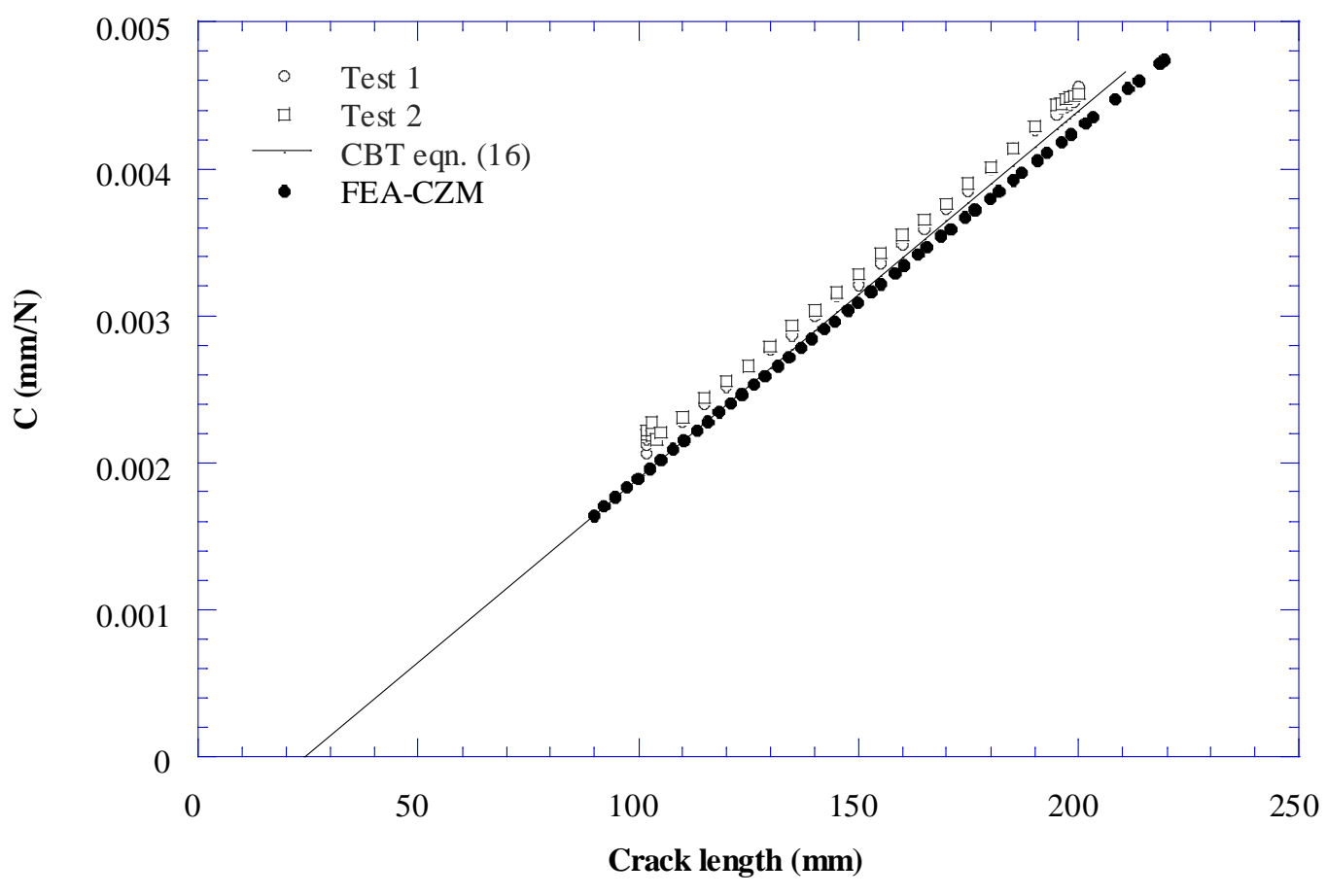

Figure 6. The variation of compliance with crack length for a tapered double cantilever beam specimen manufactured with aluminium alloy substrates and bonded with the epoxy-paste adhesive. Experimental results from two tests are shown, together with the values predicted by CBT, i.e. equation (16), and by using the finite element cohesive zone model (FE-CZM). 


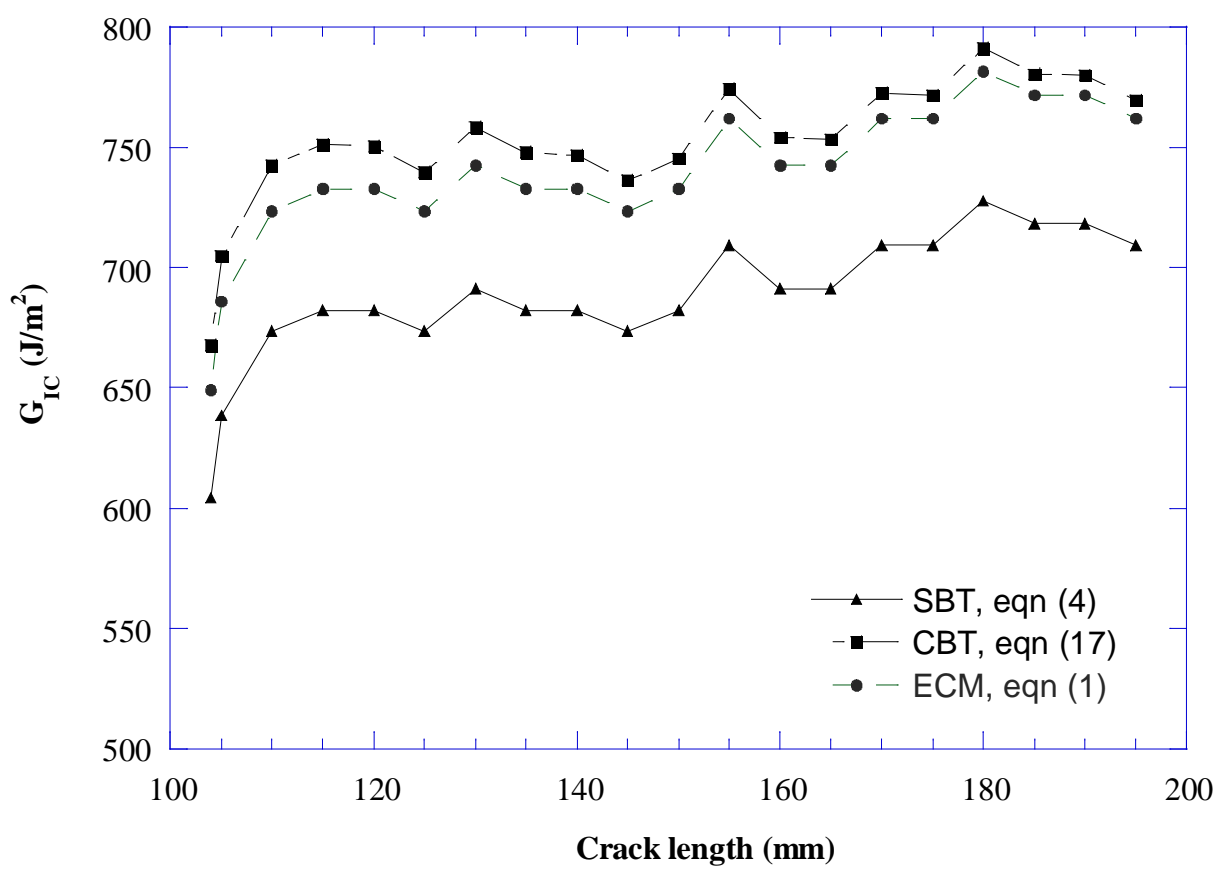

Figure 7. R-curve behaviour for a tapered double cantilever beam specimen manufactured with aluminium alloy substrates and bonded with the epoxy-paste adhesive. Values of $G_{I C}$ were deduced using; simple beam theory (SBT) i.e. equation (4), corrected beam theory (CBT), i.e. equation (17), and the experimental compliance method (ECM), i.e. equation (1). 


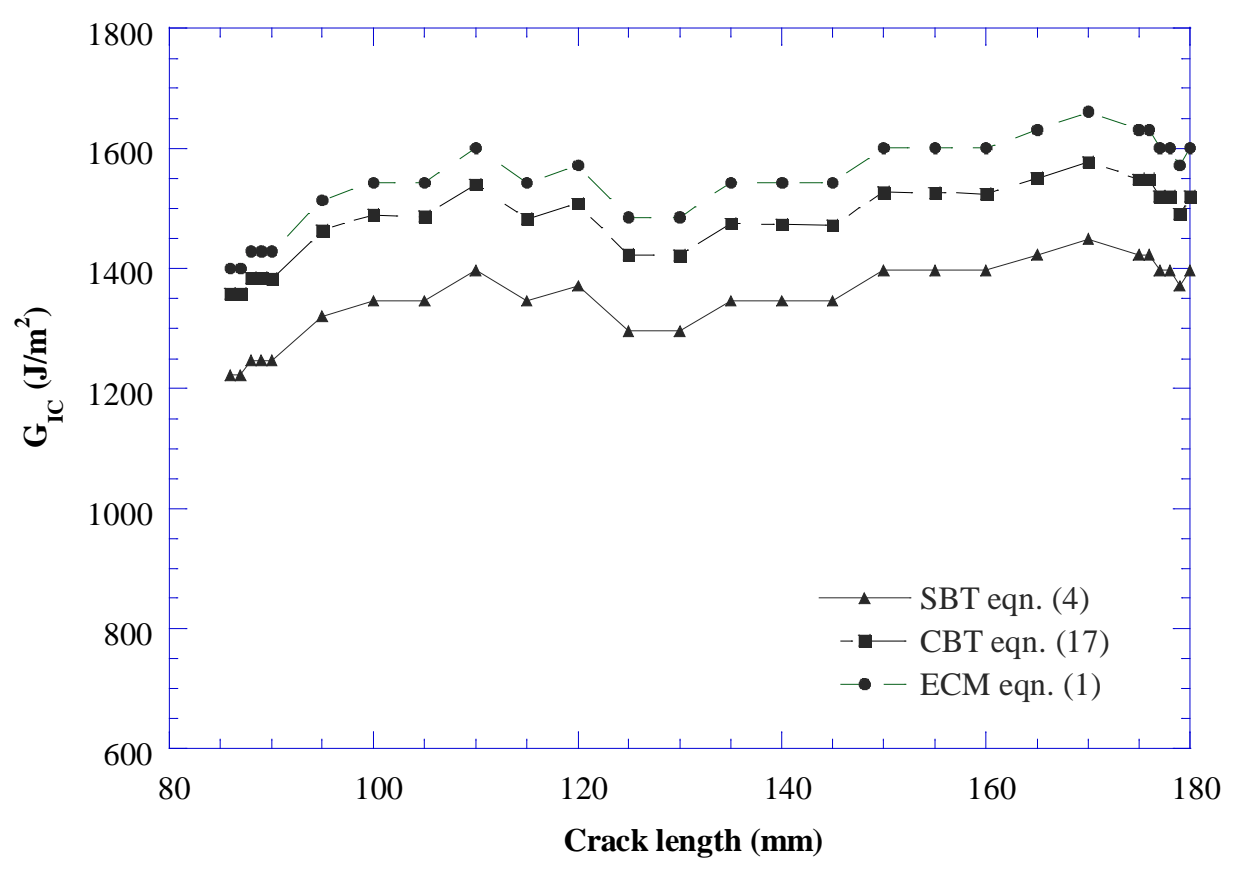

Figure 8. R-curve behaviour for a tapered double cantilever beam specimen manufactured with aluminium alloy substrates and bonded with the epoxy-film adhesive. Values of $G_{I C}$ were deduced using; simple beam theory (SBT), i.e. equation (4), corrected beam theory (CBT), i.e. equation (17), and the experimental compliance method (ECM), i.e. equation (1). 


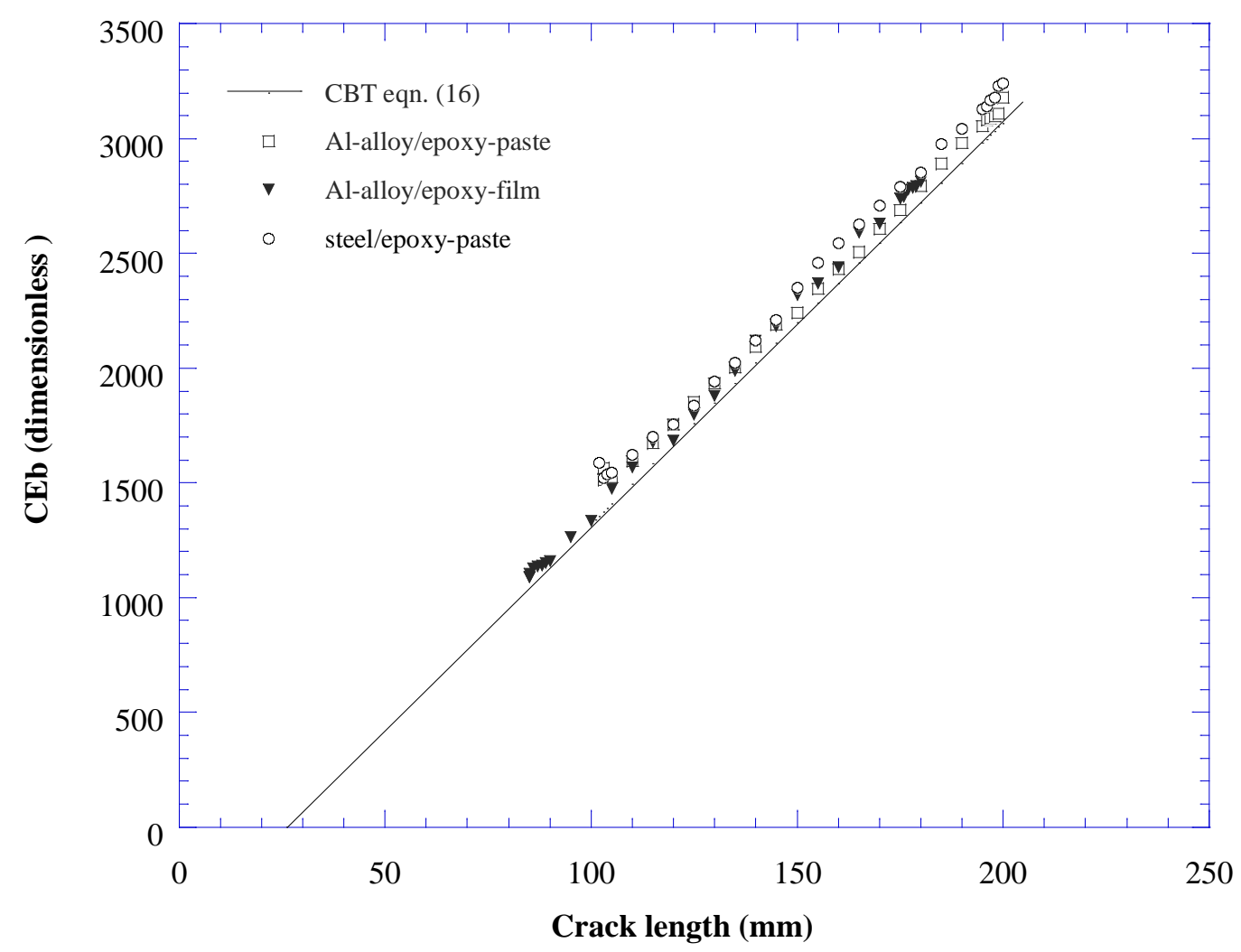

Figure 9. The variation in the values of $C E b$ with crack length for the three adhesive joint systems investigated in the present work. Experimental values are compared to the values predicted by the corrected beam theory, i.e. equation (16). 